Relato de Experiência

\title{
Avaliação da Força de Atrito em máquina de musculação durante exercícios de extensão de joelho
}

\author{
Guilherme Auler Brodt ${ }^{12}$ \\ Mônica de Oliveira Melo ${ }^{12}$ \\ Artur Bonezi ${ }^{2}$ \\ Luiz Carlos Gertz ${ }^{3}$ \\ Jefferson Fagundes Loss ${ }^{2}$ \\ ${ }^{1}$ Curso de Educação Física, Centro de Ciências da Saúde, Universidade de Caxias do Sul, \\ Caxias do Sul, RS, Brasil \\ ${ }^{2}$ Laboratório de Pesquisa do Exercício, Escola de Educação Física, Universidade Federal do Rio \\ Grande do Sul, Porto Alegre, RS, Brasil \\ ${ }^{3}$ Laboratório de Protótipos Automotivos, Engenharia Mecânica, Universidade Luterana do Brasil, \\ Canoas, RS, Brasil
}

\begin{abstract}
Resumo: Durante exercícios realizados em máquinas de musculação, a força de atrito (FA) entre seus componentes mecânicos pode influenciar na magnitude da sobrecarga oferecida pela máquina para 0 praticante do exercício. No entanto, pouco se sabe sobre como medir esse atrito e qual seu real efeito. Assim, os objetivos deste estudo foram (i) descrever uma técnica para medição da FA em máquinas de musculação, e (ii) exemplificar a técnica quantificando a FA de uma máquina de musculação durante exercício em diferentes velocidades e cargas. Foram utilizados diagramas de corpo livre, equações da mecânica clássica além da medição dos ângulos articulares com eletrogoniômetro, e da força aplicada ao cabo de aço com célula de carga, para estimar a força de atrito. Para testar a metodologia, um indivíduo realizou repetições do exercício variando cargas e velocidade de execução. As cargas avaliadas foram $3 \mathrm{~kg}$, $6 \mathrm{~kg}$ e $9 \mathrm{~kg}$; e as velocidades foram 30\%, 60\% se crescente com o incremento de velocidade e decrescente com o aumento da carga. Resultados em torno $40 \%$ obtidos para a menor carga e maior velocidade sugerem que a força de atrito deve ser um fator levado em consideração na escolha e manutenção de equipamentos de musculação.
\end{abstract}

Palavras-chave: Atrito. Musculação. Treinamento Físico.

\section{Evaluation in Resistance training machines during knee extension exercises}

\begin{abstract}
During exercises performed in weight training machines, the friction force (FF) between its mechanical components can influence the magnitude of the overload offered by the machine on force of the exercise. However, little is known about how to measure this friction and what its real effect. Thus the objectives of this study were (i) describe a technique for measuring FF in weight training machines, and (ii) exemplify the technique, by quantifying the FF of the weight training machine at different exercise speeds and loads. For this, a subject performed repetitions of the exercise in different loads and speeds. The loads evaluated were $3 \mathrm{~kg}, 6 \mathrm{~kg}$ and $9 \mathrm{~kg}$; and the speeds were $30 \% \mathrm{~s}, 60 \% \mathrm{~s}$ and $120 \% \mathrm{~s}$. The FF, normalized by selected load, increased significantly with the increasing of the speed and decreased with increasing of the load. Results for friction force around $40 \%$ found for highest speed and lowest load show that take the FF in count it's an important factor to consider during muscle equipment choice and maintaining.
\end{abstract}

Keywords: Friction. Bodybuilding. Physical Training.

\section{Introdução}

Durante a realização de exercícios físicos diversas forças agem continuamente no corpo humano, interna ou externamente, forças estas, resultantes da interação entre 0 ambiente e os corpos nele imersos (MC. GINNIS, 2002). Uma das forças presentes em nossas atividades é a força de atrito, que resulta da interação entre as moléculas de dois corpos em contato. Esta força é denominada Força de Atrito Estática - quando os corpos em contato estão em repouso - ou
Força de Atrito Dinâmica (FA) - quando os corpos em contato estão em movimento. A magnitude da força de atrito é comumente expressa pela equação 1:

$$
F A=\mu N
$$

Em que, FA é a força de atrito; $\mu$ é o coeficiente de atrito, podendo ser estático ou dinâmico e $\mathrm{N}$ é a força normal à superfície de contato

Analisando a equação (1) é possível perceber que a FA é dependente do coeficiente de atrito 
(que expressa a oposição entre as duas superfícies de contato) e da força normal. Além disso, a FA age em sentido contrário à tendência de movimento dos corpos (RADHAKRISHNAN, 1998; MC. GINNIS, 2002). Ou seja, quando o objeto está em movimento, a FA age no sentido oposto, e quando o objeto está parado, mas uma força incide sobre este objeto tentando movê-lo (ainda sem sucesso), a FA age no sentido oposto a esta força.

No contexto dos esportes e do treinamento físico, a FA também está presente em todos os movimentos e interações entre dois corpos em contato. No ciclismo, por exemplo, FA entre o solo e o pneu da bicicleta é crucial para o rendimento ideal, gerando o movimento e o equilíbrio. Por outro lado, em uma máquina de musculação, a FA pode prejudicar 0 trabalho realizado (RADHAKRISHNAN, 1998; MC. GINNIS, 2002). Máquinas de musculação têm sido recomendadas por serem práticas, seguras, de fácil familiarização e permitem a execução de exercícios de difícil realização com pesos livres (RATAMESS et al., 2009). Em uma máquina de musculação a FA é oriunda do contato entre as superfícies dos componentes mecânicos móveis da máquina de musculação, podendo estar presente no eixo de roldanas, no deslizamento dos cabos que transmitem a força e no deslocamento das pastilhas móveis da carga (PMC). Especificamente, quanto ao seu comportamento mecânico, especula-se que a FA pode influenciar na força realizada pelo indivíduo, mas não há informações referentes à sua magnitude, importância durante o treinamento, ou mesmo se é dependente de variáveis como velocidade de execução do exercício ou quantidade de carga selecionada na máquina. Muitos estudos já realizaram estimativas de força durante a realização de exercícios em máquinas de musculação, no entanto, não levaram em consideração o efeito da FA sobre o exercício. Aparelhos de remada (DE TOLEDO; DE OLIVEIRA; LOSS, 2007), extensão de joelhos (BINI et al., 2008), flexão dorsal (CANTERGI et al, 2010), entre outros são alguns dos exemplos onde $\mathrm{o}$ atrito normalmente não foi levado em consideração na estimativa de forças, tendo seu efeito subestimado.

Portanto, devido ao fato da FA possuir um efeito e importância desconhecidos quando relacionados aos equipamentos de musculação e se esse efeito pode mudar dependendo da velocidade ou da carga escolhidas, e ainda: considerando que a FA é extremamente dependente da cada máquina a partir de questões operacionais de conservação e manutenção, os objetivos deste estudo foram (i) descrever uma técnica para medição consistente da força de atrito em máquinas de musculação que utilizam pastilhas móveis para seleção da carga, e (ii) exemplificar a técnica quantificando a força de atrito de uma máquina de musculação durante exercício realizado em diferentes velocidades de execução e cargas.

\section{Métodos}

A máquina avaliada nesse estudo foi uma Mesa Romana World ${ }^{\circledR}$ (Figura 1). Essa máquina possui sistema de cabos e roldanas simétricas que transmitem a força realizada pelo indivíduo até a coluna de PMC. A PMC constitui-se por uma série de placas metálicas empilhadas, onde a quantidade de pastilhas define a carga do exercício a ser realizado. Durante o exercício de extensão de joelhos realizado nesta máquina, as cargas deslocam-se para cima (sentido ascendente) na fase concêntrica, e na fase excêntrica as cargas deslocam-se para baixo (sentido descendente), voltando à posição inicial.

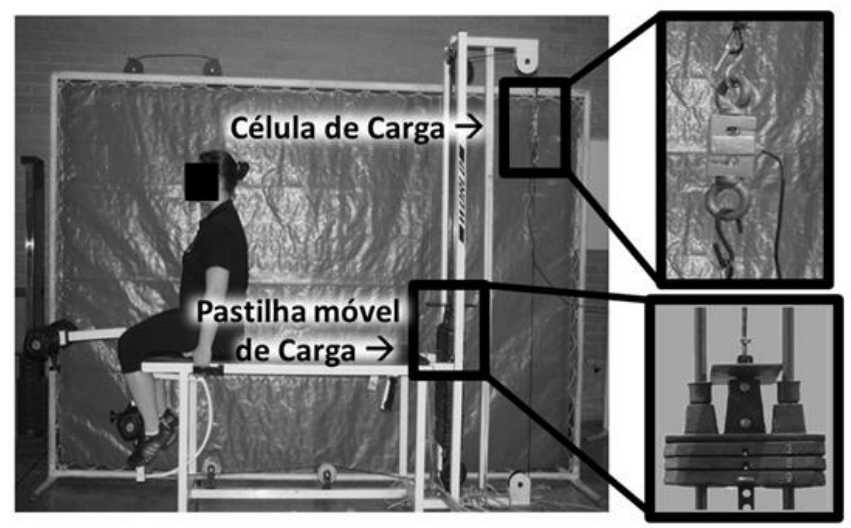

Figura 1. Máquina de musculação Mesa Romana. Em destaque a Célula de Carga acoplada e a Pastilha Móvel de Carga (PMC) guiada por duas hastes metálicas.

\section{Instrumentação}

A mesa romana foi instrumentada com uma célula de carga de $200 \mathrm{~N}$ (Alfa Instrumentos, modelo SV20) acoplada diretamente ao cabo responsável por transmitir a carga externa escolhida na PMC ao segmento envolvido no exercício (Figura 1). Um eletro-goniômetro (Miotec Equipamentos Biomédicos Ltda. ${ }^{\circledR}$ ) foi fixado diretamente ao joelho direito do indivíduo de modo que registrasse a amplitude de 
movimento (ADM) de extensão de joelho. O movimento iniciava próximos aos $90^{\circ}$ de flexão e encerrava próximo a extensão completa $\left(0^{\circ}\right.$ de flexão). Ambos os instrumentos, célula de carga e eletrogoniômetro, foram conectados a um condicionador de sinais (Miotool400 - Miotec Equipamentos Biomédicos Ltda. $\left.{ }^{\circledR}\right)$ para coleta simultânea dos dados a uma taxa de amostragem de $2000 \mathrm{~Hz}$.

\section{Análise Mecânica}

Em uma máquina de musculação há diversos locais onde o efeito das forças de atrito pode ser identificado como: o eixo das roldanas, o eixo de estruturas rígidas que se movimentam para transmitir força e torque, ou nas colunas-guias que conduzem o movimento da PMC. Mesmo em máquinas em bom estado de conservação, com manutenção e lubrificação adequadas, 0 atrito nas colunas-guias tende a ser bem maior que as demais fontes de atrito. Um esquema das principais forças atuantes durante o exercício de extensão de joelho realizado na máquina mesa romana é apresentado na Figura 2.

Ao executar o exercício analisado o indivíduo realiza uma força muscular (FM) que estende 0 joelho fazendo com que a perna empurre a parte móvel da máquina gerando uma força de contato contra a almofada de apoio, denominada força humana $(\mathrm{FH})$. A parte móvel da máquina traciona o cabo de aço transmitindo ao cabo uma força (FC), força esta que por sua vez é transmitida através das roldanas até a pastilha móvel da carga (PMC) fazendo com que a coluna de pesos se movimente. A PMC sofre assim ação de três forças principais: do cabo de aço $(F C)$, do atrito através do contato com a coluna-guia $(F A)$ e o próprio peso da coluna $(\mathrm{P})$.

Para analise do movimento da PMC (Figura 3), o exercício foi dividido em duas fases. De acordo com o gesto do indivíduo, a primeira fase, denominada fase ascendente, a PMC se move para cima ocorre quando o indivíduo estende 0 joelho (contração concêntrica da musculatura extensora de joelho). Já a segunda fase denominada fase descendente, em que a PMC se move para baixo, ocorre quando o indivíduo flexiona 0 joelho (contração excêntrica da musculatura extensora de joelho) retornando à posição inicial. Dois diagramas de corpo livre (WINTER, 2005) foram estruturados para representar todas as forças que atuam na PMC nestas duas fases, um para a fase ascendente

(Figura $3 \mathrm{~A}$ ) outro para a fase descendente (Figura $3 B$ ). As forças atuantes sobre a PMC foram representadas de acordo com o ponto de atuação considerado e sua linha de ação (LOSS; CANDOTTI, 2008).

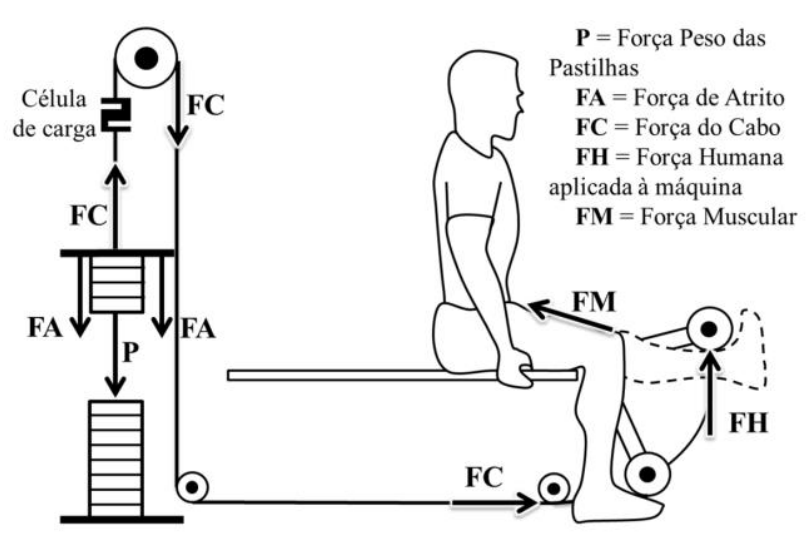

Figura 2. Esquema representativo das principais forças atuantes durante a fase ascendente da carga em um exercício de extensão de joelhos realizado na máquina de musculação Mesa Romana.

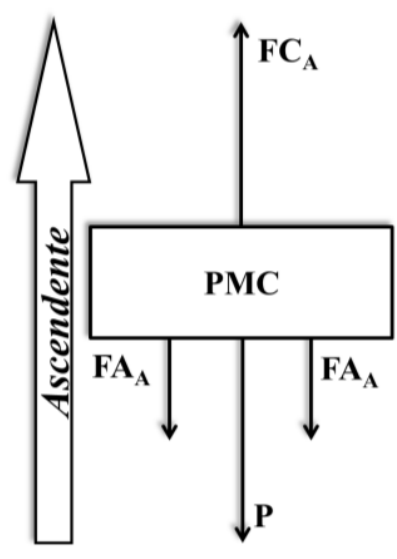

$\mathrm{FA}_{\mathbf{A}}$ - Força de Atrito Ascendente $\mathrm{FA}_{\mathrm{D}}$ - Força de Atrito Descendente D - Pastilha Móvel de Carga (A) Pase ascendente Mável de Carga \begin{tabular}{l|l} 
(A) Fase ascendente & (B) Fase descendente
\end{tabular}

Figura 3. Diagrama de corpo livre da pastilha móvel de carga (PMC) da máquina de musculação e suas forças atuantes representadas por setas pretas. A direção do movimento da PMC é representada por setas brancas largas.

Durante a fase ascendente a FA, por ser contrária ao movimento, aponta para baixo (Figura 3A). Assim sendo, nesta fase, FA será contrária à força do cabo $(F C)$. Então, quanto maior a FA entre os componentes da máquina, maior será a força necessária para movimentar a PMC durante esta fase do exercício (extensão de 
joelhos). Já na fase descendente (Figura 3B) 0 sentido da FA é para cima, acompanhando a força do cabo. Assim, nesta fase, a FA se soma à força do cabo diminuindo a força humana necessária para movimentar a PMC. Então, durante a fase de flexão de joelho, quanto maior a FA, menor será a força necessária para movimentar a PMC.

A força do cabo necessária para movimentar a PMC será medida pela célula de carga acoplada diretamente ao cabo que guia o movimento da PMC. Com base nos Diagramas de Corpo Livre (Figura 3), a situação foi descrita pela equação (2) que orquestra o movimento:

$$
\Sigma \mathrm{F}=\mathrm{m} . \mathrm{a}
$$

Onde:

$\boldsymbol{\Sigma} \mathbf{F}$ se refere à soma de todas as forcas agindo na pastilha móvel de carga

m se refere à massa da pastilha móvel de carga

a é a aceleração linear do centro de massa da pastilha móvel de carga

\begin{tabular}{|l|l|}
\hline \multicolumn{1}{|c|}{ Fase ascendente } & \multicolumn{1}{|c|}{ Fase descendente } \\
$\Sigma F=m \cdot a$ & \\
$F C_{A}-P-F A_{A}=m \cdot a_{A}$ & $F C_{D}-P+F A_{D}=m \cdot a_{D}$ \\
$P=\left(F C_{A^{-}} F A_{A}\right)-m \cdot a_{A} \quad(2.1)$ & $P=\left(F C_{D}+F A_{D}\right)-m \cdot a_{D} \quad$ (2.2) \\
\hline
\end{tabular}

Onde:

$\mathbf{a}_{\mathrm{A}}$ e $\mathbf{a}_{\mathrm{D}}$ é a aceleração linear do centro de massa da pastilha móvel de carga durante a fase ascendente e descendente, respectivamente.

Uma vez que o Peso da PMC é o mesmo nas duas fases pode-se igualar as equações $2.1 \mathrm{e}$ 2.2, resultando na equação 3 :

$$
F C_{A}-F A_{A}-m \cdot a_{A}=F C_{D}+F A_{D}-m \cdot a_{D}
$$

Considerando que a aceleração durante as fases ascendentes $\left(a_{A}\right)$ e descentes $\left(a_{D}\right)$ seja a mesma, já que o exercício foi executado com auxílio de metrônomo, e considerando ainda que força de atrito também seja a mesma durante as fases, a equação 3 pode ser re-escrita, resultando na equação 4 , onde FA representa a força de atrito em ambas as situações:

$$
\mathrm{FA}=\frac{F C A_{\mathrm{A}}-F C_{D}}{2}
$$

A partir da análise realizada com auxílio dos diagramas de corpo livre e das equações dinâmicas, pode-se constatar que a FA age em sentidos opostos em cada fase. Portanto, a partir da equação (4), diferenças encontradas nos valores da força do cabo média entre as fases, ascendente e descendente, podem ser consideradas fruto direto da FA da bateria de carga com o eixo em torno da qual ela se movimenta e de seus componentes mecânicos.

\section{Protocolo}

O protocolo de avaliação consistiu da realização de 10 repetições em 3 cargas e 3 velocidades do exercício de extensão de joelho na máquina mesa romana, totalizando 90 repetições do exercício analisadas. As repetições foram realizadas por um indivíduo treinado que assinou o termo de consentimento informado o qual foi aprovado pelo parecer 2007752 emitido pelo Comitê de Ética em Pesquisa da UFRGS onde foi realizado.

As cargas selecionadas foram de 3,6 e $9 \mathrm{~kg}$, por serem cargas de fácil execução. $O$ ritmo de execução foi controlado com um metrônomo sendo as velocidades realizadas de $30 \% \mathrm{~s}, 60 \%$ e $120 \%$ s, por serem consideradas velocidades tradicionalmente utilizadas na execução de exercícios de musculação e classificadas como lenta, moderada e rápida (KOMl; KANEKO; AURA, 1987; KRAEMER; RATAMESS, 2004). Os ritmos selecionados no metrônomo foram de 20 , 40 e $80 \mathrm{bmp}$, respectivamente, considerando uma amplitude de $90^{\circ}$ para a execução do exercício. Todas as séries foram realizadas por um mesmo indivíduo, praticante de exercícios em equipamentos de musculação a mais de dois anos, em ordem aleatória e foi realizado um intervalo de 5 minutos entre as séries a fim de evitar efeitos da fadiga (CHEN; REN, 2010). Por se tratar de uma análise puramente mecânica do equipamento, independentemente da quantidade de indivíduos que realizassem o exercício, entende-se que a máquina iria se comportar da mesma maneira, desde que mantidos os parâmetros de carga e velocidade especificados.

\section{Tratamento dos dados}

Os dados de força oriundos da célula de carga e os dados de ângulo da articulação do joelho, oriundos do eletrogoniometro foram filtrados com um filtro digital Butterworth, passa-baixa, com frequência de corte de $5 \mathrm{~Hz}$ e $3 \mathrm{~Hz}$ respectivamente, definidas pelo critério de resíduos de Winter (WINTER, 2005). Para cada fase de cada uma das 90 execuções realizadas foram computados os valores médios da força do cabo (FC) medido pela célula de carga. O valor da FA foi calculado segundo a equação (4), em valores absolutos expresso em kgf, e também em valores percentuais, dividindo o valor obtido pela carga utilizada para realização de cada exercício. 
O tratamento estatístico foi realizado por meio do software SPSS $^{\circledR}$ versão 10.0, onde foi confirmada a equivalência das variâncias inicialmente com 0 teste de Levene, e a normalidade dos dados com o teste de ShapiroWilk. Para verificar o efeito dos fatores carga e velocidade foi utilizada uma ANOVA two-way de medidas repetidas, e para identificar onde estavam as diferenças foi usado um teste posthoc com ajustes de Bonferroni. O nível de significância de $\alpha \leq 0,05$ foi adotado para todas as análises estatísticas.

\section{Resultados}

A Figura 4 exemplifica a força do cabo e o deslocamento angular do joelho ao longo do tempo durante uma execução do exercício a $60^{\circ}$ por segundo com a carga de $3 \mathrm{~kg}$. Pode-se perceber que os valores de força adquiridos pela célula de carga durante a fase ascendente são sensivelmente maiores que os adquiridos durante a fase descendente nesta situação.

A Figura 5 apresenta os resultados dos efeitos principais da FA, em valores absolutos para todas as variáveis testadas. É possível perceber que a velocidade contribuiu significativamente para 0 aumento da FA. À medida que a velocidade aumenta a FA também aumenta, apresentando valores crescentes em média $1,04( \pm 0,18) \mathrm{kgf}$ na velocidade de $30 \% \mathrm{~s}, 1,45( \pm 0,29) \mathrm{kgf}$ para $60 \% \mathrm{~s} \mathrm{e}$ $2,53( \pm 0,61) \mathrm{kgf}$ para $120 \%$ s. Já na comparação entre as cargas testadas, foram encontrados resultados de $1,40( \pm 0,59) \mathrm{kgf}$ de FA para a carga de $3 \mathrm{~kg}, 1,82( \pm 0,74) \mathrm{kgf}$ para $6 \mathrm{~kg}$ e $1,90( \pm 0,82)$ $\mathrm{kgf}$ para a carga de $9 \mathrm{~kg}$. Ainda, de acordo com

\section{Velocidades}

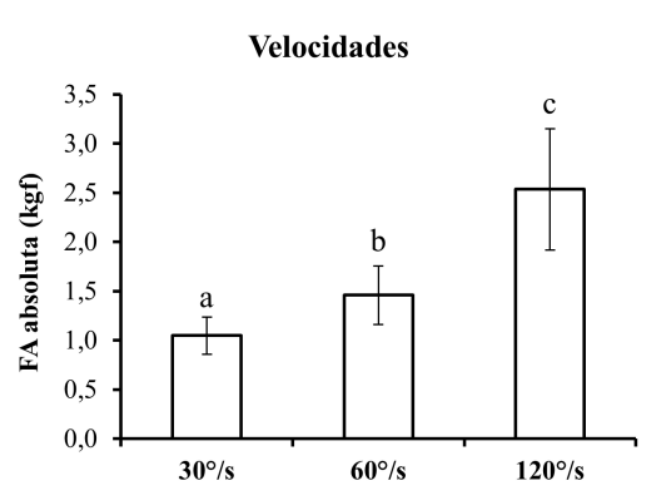

os resultados supra escritos a FA aumenta significativamente com incremento na carga de 3 para 6 kgf, mas não de 6 para 9 kgf.

Quando a FA é relativizada pela carga escolhida, o mesmo comportamento crescente foi encontrado na analise do fator velocidade, mas um comportamento decrescente foi obtido para o fator carga (Figura 6). Para a velocidade de $30 \% \mathrm{~s}$ a FA representou $11,8 \%( \pm 1,9)$ da carga medida, $16,8 \%( \pm 3,1)$ para $60 \%$ s e $29,2 \%( \pm 7,6)$ para a velocidade de $120 \%$ s. A FA relativa à carga escolhida apresentou um comportamento decrescente conforme foram aumentadas as cargas, resultando em $46,7 \%( \pm 19,7)$ da carga de $3 \mathrm{~kg}, 30,3 \%( \pm 12,3)$ para a carga de $6 \mathrm{~kg}$ e $21,1 \%$ $( \pm 9,1)$ para a carga de $9 \mathrm{~kg}$, demonstrando que, aparentemente, sua importância sobre a força humana total diminui à medida que se escolhem cargas maiores.

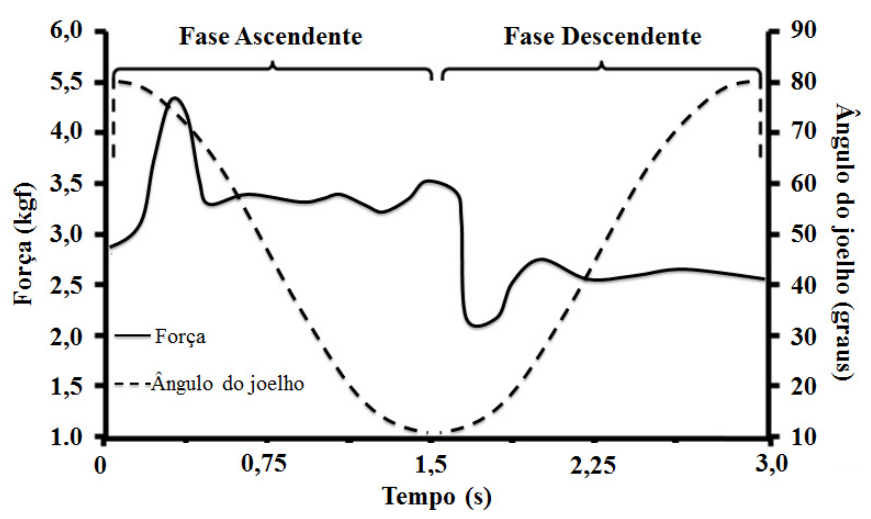

Figura 4. Gráfico representativo do comportamento da força do cabo (FC), medido pela célula de carga, e do ângulo da articulação do joelho ao longo tempo de uma execução completa, com uma carga de $3 \mathrm{~kg}$, em uma velocidade de $60 \%$ s.

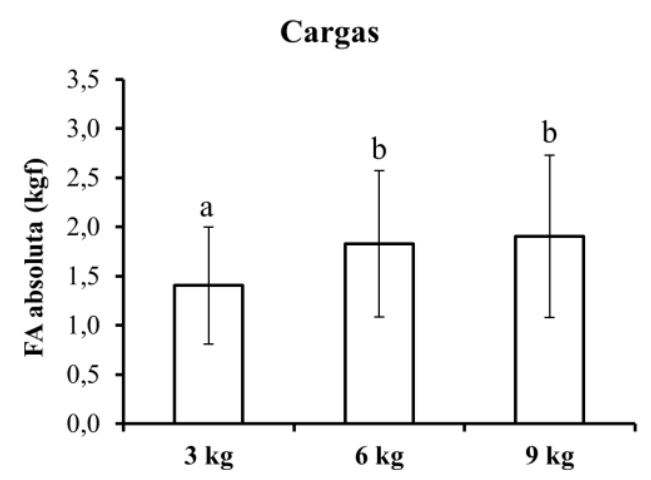

Figura 5. Resultados da comparação da força de atrito durante as três velocidades e três cargas, em valores absolutos. Letras diferentes indicam diferenças estatisticamente significativas $(p<0,05)$. 

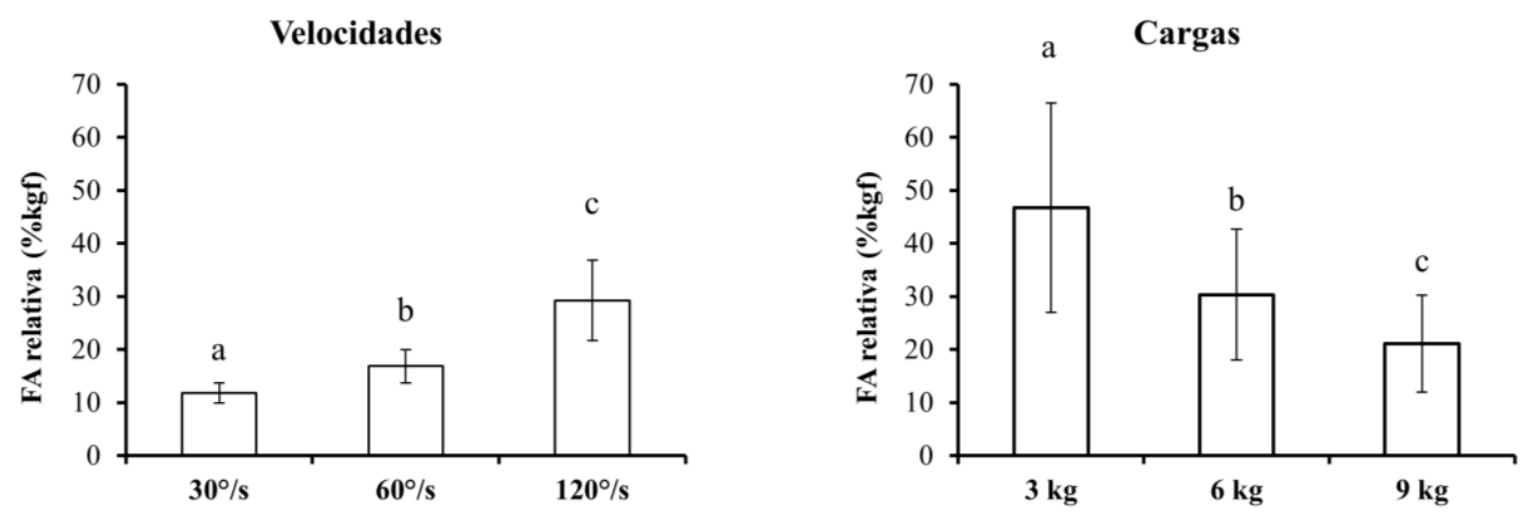

Figura 6. Resultados da comparação da FA relativa durante as três velocidades e três cargas, em valores percentuais relativos à carga utilizada em cada exercício. Letras diferentes indicam diferenças estatisticamente significativas $(p<0,05)$.

\section{Discussão}

Um dos objetivos deste estudo foi, primeiramente, descrever um procedimento para estimar de maneira consistente a FA a partir de procedimentos experimentais. Para isso foi necessário instrumentar a máquina de musculação com uma célula de carga no cabo, entre a PMC e o apoio para a perna, onde 0 executante aplica força. $O$ procedimento proposto é relativamente simples, mas exige que todos os passos sejam seguidos. Uma análise mecânica da situação através dos diagramas de corpo livre e das equações do movimento da PMC aponta que basta possuir as informações da força do cabo durante a execução de exercícios executados em ritmos simétricos (mesmo tempo durante a movimentação ascendente $e$ descendente da PMC) para se obter a FA (equação 4).

Dentro da variabilidade de cargas e velocidades analisadas, foram encontrados valores de aproximadamente $1,0 \mathrm{kgf}$ a $2,5 \mathrm{kgf}$ para a FA durante os testes realizados. Os maiores valores de FA foram obtidos em altas cargas e altas velocidades. Os valores da FA variaram de aproximadamente $10 \%$ a $40 \%$ da carga utilizada, chamando a atenção para a importância de se considerar os efeitos da FA durante a execução dos exercícios, especialmente em exercícios executados com baixa carga. Certamente estes resultados não podem ser extrapolados para outros equipamentos, mas na medida em que se podem ter forças de atrito correspondendo a mais de $40 \%$ da carga selecionada em uma determinada máquina, é razoável supor inclusive que valores maiores podem ser encontrados em outros equipamentos, principalmente se houverem problemas com a manutenção e lubrificação da máquina. Neste sentido, o detalhamento do procedimento aqui aplicado permite que usuários possam reproduzi-lo em equipamentos específicos, avaliando a força de atrito em sua própria situação.

Como a FA possui o comportamento de ser contrária a tendência de movimento da PMC, nas situações analisadas a força necessária para movimentar a PMC poderia ser de $10 \%$ até $40 \%$ maior durante a fase concêntrica (fase ascendente da PMC) e de 10 a $40 \%$ menor na fase excêntrica (fase descendente da PMC). Sob o ponto de vista do treinamento físico essas alterações podem afetar diretamente no controle da magnitude da carga de treino selecionada.

Conforme o que é usualmente encontrado na literatura, o atrito entre sólidos é uma força que age paralela à superfície e que não depende nem da área de contato entre os corpos sólidos em questão e nem da velocidade os quais estes corpos se movimentam (RADHAKRISHNAN, 1998; MC. GINNIS, 2002). No entanto, foram encontrados aumentos da FA concomitantemente ao aumento da velocidade. Isso pode ter ocorrido porque 0 atrito em atuadores mecânicos desta natureza é devido principalmente à viscosidade ${ }^{1}$ do fluido utilizado para lubrifica-los - neste caso a

\footnotetext{
${ }^{1}$ Entende-se viscosidade como uma medida da resistência à deformação de um fluido que está sendo deformado por uma tensão de cisalhamento ou tensão de tração. A viscosidade representa o "atrito interno" de um fluido, ou seja, quanto menos viscoso o fluido, maior é a sua facilidade de circulação (fluidez) ou menor é a dificuldade de deformá-lo. Exemplificando, a água é um fluído de baixa viscosidade, enquanto que o mel tem uma alta viscosidade. (SYMON, 1971).
} 
PMC da máquina de musculação (Figura 1) localizado na barra de ferro, ou trilho, o qual guia o movimento da PMC, e devem ser levadas em consideração as propriedades dos fluidos Newtonianos.

De acordo com as leis dos fluidos newtonianos, quanto maior a velocidade do corpo (neste caso o corpo em questão é a PMC ilustrada na Figura 7), que se movimenta sobre 0 fluido, maior será à força de cisalhamento entre o fluido e os corpos, devido ao gradiente transversal de velocidade (FOX; MCDONALD; PRITCHARD, 1998). Ou seja, como o atrito viscoso é dependente da velocidade (DIETER; BACON, 1986; DILDA et al., 2007), quanto maior a velocidade maior será a FA entre os corpos. No entanto, esse aumento na FA não aumenta na mesma proporção quando se trata do aumento da carga, como ilustram os resultados da Figura 6 onde a FA relativa à carga diminui com o aumento da velocidade.

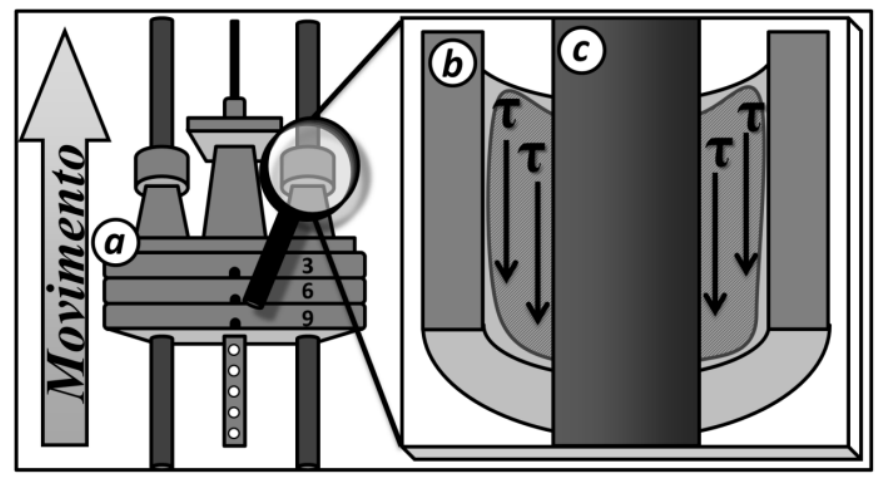

Figura 7. Imagem esquemática: a letra (a) indica a Pastilha Móvel de Carga, a seta à extrema esquerda indica o sentido e a direção do seu movimento; $(\boldsymbol{b})$ indica a guia que orienta esse movimento no trilho, o qual é indicado pela letra $(\boldsymbol{c})$. O zoom ilustra a interação entre o fluido lubrificante (área sombreada) e os componentes da máquina, $\boldsymbol{\tau}$ indica a tensão de cisalhamento procedente da viscosidade do fluido, esses componentes ( $\boldsymbol{b}$ e $\boldsymbol{c}$ ) se mantêm muito próximos (cerca de $0,3 \mathrm{~cm}$ ) e a quantidade de fluído lubrificante é bastante pequena, o que a torna sua ação também pequena, porém não desprezível. De acordo com as leis dos fluidos newtonianos, quanto maior a velocidade do corpo (neste caso o corpo em questão é o $\boldsymbol{b}$ ) que se movimenta sobre o fluido maior será à força de cisalhamento entre o fluido e os corpos.

\section{Limitações}

O controle do ritmo da velocidade com o metrônomo, mesmo sendo muito criterioso e garantindo o intervalo de tempo de cada fase do exercício, não é capaz de controlar a velocidade da execução, assim o exercício pode apresentar uma velocidade variável durante a execução mesmo tendo uma velocidade média controlada. Por outro lado, após uma variabilidade inicial nos primeiros graus da amplitude de movimento, é possível perceber uma estabilização dos valores da força do cabo, mensurada pela célula de carga (Figura 4). Esta manutenção dos valores da curva de força sugere um movimento com uma variação de velocidade muito baixa (aceleração desprezível). Estes resultados tornam menos crítica a equiparação dos valores de aceleração durante as fases ascendente e descendente, conforme foi sugerido na equação 3 da metodologia. Outro aspecto está relacionado a gama de cargas e velocidades analisadas. A utilização de apenas três cargas e três velocidades não permite a extrapolação para situações além daquelas avaliadas.

\section{Repercussão Prática}

$O$ fato de a FA diminuir a carga externa durante a fase descendente da PMC, fase na qual ocorre a contração excêntrica da musculatura extensora de quadril, vai de encontro ao princípio do treinamento que diz respeito à sobrecarga (WEINECK, 1999), que postula que para atingir os ganhos fisiológicos ótimos do treinamento de força e hipertrofia o exercício deverá ser realizado com a sobrecarga máxima para o número de repetições pretendidas - repetições máximas ou RMs - (KRAEMER; RATAMESS, 2004; RATAMESS et al., 2009) ou sobrecarga a qual o indivíduo não está habituado (WEINECK, 1999). Então, uma vez que durante a fase excêntrica possuímos a maior capacidade de produção de força por unidade muscular e, além disso, um maior recrutamento muscular (KOMl; KANEKO; AURA, 1987; ENOKA, 1996), seria durante a fase 
excêntrica que deveríamos ter a maior carga externa, e consequentemente maior força humana necessária para a realização do exercício, para assim 0 treinamento trazer melhores ganhos (SEGER; ARVIDSSON; THORSTENSSON, 1998; FARTHING; CHILIBECK, 2003). Cabe salientar que as maiores forças de atrito (em valores absolutos) foram encontradas nas maiores velocidades e nas maiores cargas. Porém, quando analisada percentualmente, a força de atrito cai bastante com o aumento da carga, ou seja, em baixas cargas (e altas velocidades) a força de atrito compõe parte importante da carga, chegando, neste caso, a $40 \%$ da carga escolhida, enquanto que com altas cargas (e baixas velocidades) a força de atrito ficou próxima a $10 \%$ da carga escolhida, demonstrando que sua importância diminuiu com o aumento da carga.

No entanto, é importante chamar a atenção que este método está restrito às condições experimentais: máquinas de musculação semelhantes a esta, com sistema de cabos que transmitem a força realizada pelo indivíduo conectados por cabos diretamente à PMC, que se movimente em colunas-guia. Sugere-se utilizar o método com outros tipos de máquina bem como em uma faixa mais ampla de cargas.

\section{Conclusão}

Ao verificar a interferência da FA em uma mesa romana, buscou-se, além da quantificação da força de atrito, a especificação de um método que pode ser reproduzido com facilidade. Percebeu-se que a FA, expressa em valores percentuais, é dependente tanto da velocidade (relação direta) quanto da quantidade de carga (relação inversa) utilizada para realização dos exercícios, agindo de forma a criar uma diferença entre a fase excêntrica e concêntrica que, dentro da faixa observada, pode chegar a mais de $40 \%$ da carga selecionada. Portanto, destaca-se a importância da verificação periódica dos componentes das máquinas de musculação pelos proprietários de academias e de máquinas de musculação e da qualidade dos equipamentos utilizados.

\section{Referências}

BINI, R.R.; CARPES, F.P.; TOLEDO, J.M.D.; LOSS, J.F. Estimativa das forças na articulação tíbio-femoral no exercício de extensão dos joelhos em cadeia cinética aberta realizado em máquina de musculação. Revista Brasileira de Cineantropometria \& Desempenho Humano, Florianópolis, v.10, p.35-42, 2008.

CANTERGI, D; ABECH, E; GOMES, L.E.; LOSS, J.F. Análise mecânica e estimativa da força muscular resultante no aparelho flexor dorsal do pé. Revista Brasileira de Ciência e Movimento, Taguatinga, v. 18, n. 1, p. 11-18, 2010

CHEN, L.; REN, L. The influence of intrinsic muscle properties on musculoskeletal system stability: A modelling study. Journal of Bionic Engineering, Changchun, v.7, n.SUPPL., 2010.

DE TOLEDO, J.M.; DE OLIVEIRA, P.R.C.; LOSS, J.F. Análise biomecânica de um aparelho de remada. Anais do XII Congresso Brasileiro de Biomecânica. São Pedro: UNESP, p.868-873, 2007.

DIETER, G.; BACON, D. Metallurgy and Mechanical Engeneering. ed.:McGraw-Hill, New York, v.45. 1986

DILDA, V.; MIOTTO, F.; VALDIERO, A.; ANDRIGHETTO, P.; RAFIKOV, M. Estudo do Comportamento Dinâmico de um Atuador Hidráulico e suas Características Não Lineares.Proceedings of the Brazilian Conference on Dynamics, Control and Their Applications (DINCON). São José do Rio Preto-SP, Brazil, 2007. p. 1-7

ENOKA, R.M. Eccentric contractions require unique activation strategies by the nervous system. Journal of Applied Physiology, Bethesda, v.81, n.6, p.2339-2346, 1996.

FARTHING, J.P.; CHILIBECK, P.D. The effects of eccentric and concentric training at different velocities on muscle hypertrophy. European Journal of Applied Physiology, Berlin, v.89, n.6. p.578-586, 2003.

FOX, R.W.; MCDONALD, A.T.; PRITCHARD, P.J. Introdução à Mecânica dos Fluidos. $6^{\mathrm{a}}$ ed. :LTC Editora, Rio de Janeiro, v.6. 1998

KOMI, P.V.; KANEKO, M.; AURA, O. EMG activity of the leg extensor muscles with special reference to mechanical efficiency in concentric and eccentric exercise. International Journal of Sports Medicine, Stuttgart, v.8, n.SUPPL. 1, p.22-29, 1987.

KRAEMER, W.J.; RATAMESS, N.A. Fundamentals of Resistance Training: Progression and Exercise Prescription. Medicine and Science in Sports and Exercise, Indianapolis, v.36, n.4, p.674-688, 2004. 
LOSS, J.F.; CANDOTTI, C. Comparative study between two elbow flexion exercises using the estimated resultant muscle force. Revista

Brasileira de Fisioterapia, São Carlos, v.12, p.502-510, 2008.

MC. GINNIS, P.M. Biomecânica do esporte e exercício. $1^{\mathfrak{a}^{\mathrm{a}}}$ ed.:Artmed, Porto Alegre. 2002

RADHAKRISHNAN, V. Locomotion: Dealing with friction. Proceedings of the National Academy of Sciences, Washington, v.95, n.10, p.54485455, 1998.

RATAMESS, N.A.; ALVAR, B.A.; EVETOCH, T.K.; HOUSH, T.J.; KIBLER, W.B.; KRAEMER, W.J.; TRIPLETT, N.T. Progression models in resistance training for healthy adults. Medicine and Science in Sports and Exercise, Indianapolis, v.41, n.3, p.687-708, 2009.

REMAUD, A.; GUÉVEL, A.; CORNU, C. Antagonist muscle coactivation and muscle inhibition: effects on external torque regulation and resistance training-induced adaptations.

Neurophysiologie Clinique/Clinical

Neurophysiology, Louvain-La-Neuve, v.37, n.1, p.1-14, 2007.

SEGER, J.Y.; ARVIDSSON, B.;

THORSTENSSON, A. Specific effects of eccentric and concentric training on muscle strength and morphology in humans. European Journal of Applied Physiology and Occupational Physiology, Berlin,v.79, n.1, p.49-57 1998.

SYMON, K. Mechanics. ed.:Addison Wesley. , Massachusetts, v.3. 1971.639p.

WEINECK, J. Treinamento ideal instruções técnicas sobre o desempenho fisiológico, incluindo considerações específicas de treinamento infantil e juvenil. ed.:Manole Ltda, São Paulo, v.9. 1999

WINTER, D. Biomechanics and Motor Control of Human Movement. $3^{\mathrm{a}}$ ed. : John Wiley \& Sons, New Jersey. 2005

\section{Endereço:}

Guilherme Auler Brodt

Rua Felizardo, 750 Bairro: Jardim Botânico

Porto Alegre RS Brasil

90690-200

Telefone: (51) 3308-5822

e-mail: guilhermebrodt@gmail.com

Recebido em: 30 de abril de 2012.

Aceito em: 16 de abril de 2013.

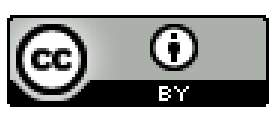

Motriz. Revista de Educação Física. UNESP, Rio Claro, SP, Brasil - elSSN: 1980-6574 - está licenciada sob Creative Commons - Atribuição 3.0 